\section{Patricia Moya ${ }^{1}$ Eduardo Chappuzeau ${ }^{2}$ Juan Carlos Caro $^{3}$ María José Monsalves ${ }^{4}$}

Master en Salud Pública e Institu ciones de Salud. Profesor de Salud Pública e Investigación. Facultad de Odontología, Universidad Finis Terrae. Santiago de Chile, Chile.

2 Cirujano-Dentista. Universidad Finis Terrae. Santiago de Chile, Chile.

${ }^{3}$ Master en Salud Pública e Instituciones de Salud. Profesor de Salud Pública e Investigación. Facultad de Odontología, Universidad Finis Terrae. Santiago de Chile, Chile.

4 Magister en Epidemiología Clínica Profesor de Salud Pública e Investigación. Facultad de Odontología Universidad Finis Terrae. Santiago de Chile, Chile.

\section{Correspondencia:}

Dra. Patricia Moya Rivera. Facultad de Odontología Universidad Finis Terrae Av. Pedro de Valdivia 1505 Providencia, Santiago de Chile. E-mail: drapatriciamoya@yahoo.com

\title{
Situación de salud oral y calidad de vida de los adultos mayores
}

Moya $P^{1}$, Chappuzeau $E^{2}$, Caro JC ${ }^{3}$, Monsalves $M J^{4}$. Situación de salud oral y calidad de vida de los adultos mayores. Rev Estomatol Herediana. 2012; oct-dic 22(4):197-202.

\section{RESUMEN}

Objetivos: Conocer la situación de salud oral y calidad de vida del adulto mayor que asiste a centros de salud pública del área oriente de Santiago de Chile durante los años 2011-2012. Material y métodos: Estudio descriptivo de corte transversal en una muestra probabilística de $\mathbf{3 8 0}$ adultos mayores. Se evaluó historia de caries, estado periodontal y situación protésica. La percepción de calidad de vida se midió con el Geriatric Oral Health Assessment Index (GOHAI). Se establecieron dos categorías: "mala calidad de vida" (puntuación $\leq 57$ ) y "buena calidad de vida" (puntuación $>57$ ). El análisis estadístico se hizo en programa STATA 11.0. Resultados: La edad media fue de 71,33 años (D.E. $\pm 7,26$ ); $66,1 \%$ son mujeres; $51,6 \%$ casado y $78,9 \%$ pensionado. Un $32,4 \%$ tiene educación media completa. El COPD fue de 22,16 (IC: 95\% 21,72-22,60) mayor en mujeres $(\mathrm{p}>0,05)$ y aumenta con la edad $(\mathrm{p}<0.05)$. Un $44 \%$ tiene entre 1 y 4 piezas dentarias con lesiones de caries dental. El edentulismo fue de 7,1\%. Un $20 \%$ presentó sacos periodontales $>$ a $5,5 \mathrm{~mm}$. Un 43\% presenta higiene oral regular. Un 59,5\% usa prótesis removible en el maxilar superior y $40,3 \%$ en el inferior. Un 76,6\% percibe como mala su calidad de vida. Conclusión: Los adultos mayores son un grupo de especial interés para la salud pública debido a la mala percepción de su calidad de vida y vulnerabilidad de su salud bucal.

Palabras clave: ADULTO MAYOR, SALUD ORAL, CALIDAD DE VIDA.

Oral health status and quality of life of older adults.

\section{ABSTRACT}

Objectives: The purpose of this study was to determine de the status of oral health and life quality of older adults who attend in public health centers in the area orient of Santiago, Chile during the years 20112012. Material and method: A descriptive cross-sectional study in a random sample of 380 older adults. Was evaluated the history of caries, periodontal and prosthetic situation. The perceived life quality was measured with the Geriatric Oral Health Assessment Index (GOHAI). Were established two categories: "Bad quality life" (score $\leq 57$ ) and "Good life quality" (score $>57$ ). The statistical analysis was done in STATA 11.0. Results, the average age was 71.33 years (E.D. \pm 7.26$) ; 66.1 \%$ are women; $51.6 \%$ are married $y 78.9 \%$ retired. $32.4 \%$ have completed secondary education. The CAOD was 22.16 (CI $95 \% 21.72$ to 22.60$)$ more in women $(p>0.05)$ and increased with age $(p<0.05)$. The $44 \%$ have between 1 and 4 teeth with dental caries. Edentulous was $7.1 \%$. The $20 \%$ presented periodontal pockets $>5.5 \mathrm{~mm}$. About $43 \%$ have regular oral hygiene. The $59.5 \%$ a removable prosthesis used in the maxilla and $40.3 \%$ in the mandible. The $76.6 \%$ perceived bad quality of life. Conclusion: The elderly are a group of special interest for public health due to poor perception of their life quality and oral health vulnerability.

Key words: OLDER ADULTS, ORAL HEALTH, QUALITY OF LIFE.

\section{Introducción}

En Chile, los adultos mayores tiene cada vez más peso relativo en el total de la población, alcanzando alrededor del 13\% (1). Si bien el envejecimiento es un proceso fisiológico deteriorativo asociado a una disminución de la viabilidad y aumento de la vulnerabilidad, las evidencias en el individuo comienzan a manifestarse a partir de la sexta década de la vida (2).

Con el proceso de envejecimiento se generan cambios en el estado social, percepción sensorial y en funciones cognitivas y motoras repercutiendo en las actividades del diario vivir. Se presenta elevada prevalencia de enfermedades crónicas, asociadas a desigualdades en relación a género y nivel educacional (1). La percepción sobre la salud es mala, siendo peor en las mujeres y entre quienes tienen menor nivel educacional (3).

Existe evidencia sobre la relación entre la salud bucal y la salud general, principalmente como resultado de factores de riesgo comunes entre condiciones bucales y enfermedades crónicas no transmisibles (4). Una salud bucal deficiente en adultos mayores generalmente está acompañada por una mala salud general, repercutiendo en su calidad de vida $(5,6)$.

Los adultos mayores corresponden al grupo etario que presenta mayor morbilidad bucal, como con- 
secuencia de no haber recibido durante su vida suficientes medidas de prevención o tratamientos adecuados y oportunos para recuperar su salud bucal (7). La pérdida de todos los dientes (edentulismo) está relacionada con experiencias de procesos infecciosos (no atendidos o atendidos de manera deficiente) y falta de higiene más no como una consecuencia del envejecimiento (4).

El primer estudio nacional de salud oral en adultos chilenos finalizado el año 2009 determinó que la prevalencia de enfermedad periodontal afecta a más del $90 \%$ de la población, siendo mayor el daño en los adultos mayores y en aquellos con menos de 12 años de educación(8); presentan además, una alta prevalencia de lesiones de la mucosa oral y la mayor incidencia de cáncer oral $(9,10)$.

Los adultos mayores representan un grupo de especial interés debido a la vulnerabilidad de su salud bucal $\mathrm{y}$ al acelerado crecimiento que han presentado en las últimas décadas en la mayoría de los países (11). La importancia radica principalmente en la necesidad de contar con información válida para la correcta planificación de programas asistenciales, así como también, la de generar evidencia respecto a resultados de la asistencia prestada durante todo el ciclo vital (12).

El objetivo de este estudio fue conocer la situación de salud oral y calidad de vida en los adultos mayores que asisten a los centros de salud públicos del área oriente de Santiago de Chile.

\section{Material y Métodos}

El diseño del estudio fue observacional y transversal. La población estudiada correspondió a adultos de edad igual o mayor a 60 años, beneficiarios del sistema público de salud que asisten a dos centros de salud del área oriente de Santiago de Chile. El presente estudio fue realizado en los centros de salud públicos de la comuna de Vitacura y Providencia, ambos pertenecientes al área oriente de Santiago de Chile. El marco muestral se construyó a partir de los beneficiarios validados por el Fondo Nacional de Salud (FONASA) para cada centro de salud. La muestra fue seleccionada en forma aleatoria de una lista rutificada proporcionada por los centros de salud. Se estableció como criterio de inclusión ser autovalentes, no inmunosuprimidos. El tamaño de la muestra seleccionada en cada centro de salud fue proporcional a la población asignada, siendo de un total de 380 adultos mayores.

Previa firma del consentimiento informado, se realizó un examen estandarizado siguiendo recomendaciones de la Organización Mundial de la Salud (OMS) para Encuestas de Salud Bucal. Se usó espejo plano $\mathrm{N}^{\circ} 5$ y sonda periodontal tipo OMS. El examen fue realizado en una clínica dental en cada centro de salud por un equipo de odontólogo-asistente entrenado y calibrado (Kappa 0,90). Se diseñó una ficha para el registró de sexo, edad, nivel educacional, estado civil, situación laboral. La historia de caries (índice COPD) para medir el estado de los dientes permanentes (cariados, obturados y extraídos); estado periodontal y necesidad de tratamiento aplicando el índice de necesidad de tratamiento periodontal en la comunidad (CPITN); la higiene bucal a través del Índice de Higiene Oral Simplificado (IHOS) y la situación protésica en cuanto a uso y tipo de aparato protésico en una o ambas arcadas y la necesidad de rehabilitación protésica.

Para medir la calidad de vida relacionada con la salud oral, se aplicó el índice de valoración de salud oral geriatrica (GOHAI), instrumento validado para el habla hispana. Se fundamenta en que la salud oral fue definida como ausencia de dolor e infección compatible con una dentición funcional y cómoda que permita al individuo continuar en su rol social. Según puntuación del GOHAI se establecieron dos categorías: "mala calidad de vida" (puntuación $\leq 57$ ) y "buena calidad de vida" (puntuación >57).

La base de datos se confeccionó en Excel 2007 y los datos fueron tabulados y codificados para ser transferidos al sofware STATA versión 11.0 para su análisis estadístico. Los resultados fueron estratificados por sexo y grupo de edad. Se utilizaron tablas de frecuencias, estimaciones puntuales y por intervalos de confianza del 95\%. Se calculó el nivel de significancia para la comparación del GOHAI.

Se consideró el control de la confidencialidad y anonimato de los participantes mediante el uso de folios y el resguardo de los instrumentos de recolección y de las bases de datos.

\section{Resultados}

Un $66 \%$ de la muestra fueron mujeres. La edad media fue de 71,33 años (D.E. $\pm 7,26$ ). Un $44,2 \%$ se presentó en el rango de edad entre 60 y 69 años y un 32,1\% entre 70 y 79 años, sin presentar diferencias estadísticamente significativas por sexo $(p=0,803)$. Al estudiar el nivel educacional, un 32,3\% reporta educación media completa y un $26,1 \%$ educación universitaria. En cuanto al estado civil, un $51,58 \%$ esta casado y un $13,16 \%$ viudo. 
El $57 \%$ de los adultos mayores esta pensionado y el $21,8 \%$ pensionado y con trabajo actual (Tabla 1 ).

Todos los adultos mayores presentaron historia de caries dental $y$ un $50,26 \%$ lesiones cavitadas

Tabla 1. Variables sociodemográficas de los adultos mayores. Área Oriente, Santiago de Chile, años 2011-2012.

\begin{tabular}{llcc}
\hline Variable & Categoría & $\mathbf{N}$ & $\mathbf{\%}$ \\
\hline \multirow{4}{*}{ Sexo } & Hombre & 129 & 33,95 \\
& Mujer & 251 & 66,05 \\
& Total & 380 & 100,0 \\
Grupo de edad & 60 a 69 años & 168 & 44,21 \\
& 70 a 79 años & 122 & 32,11 \\
& 80 a 89 años & 90 & 23,68 \\
\multirow{4}{*}{ Nivel educacional } & Educación básica & 93 & 24,47 \\
& Educación media & 123 & 32,37 \\
& Educación técnica & 65 & 17,11 \\
& Educ. universitaria & 99 & 26,05 \\
\multirow{5}{*}{ Estado civil } & Soltero & 66 & 17,37 \\
& Casado & 196 & 51,58 \\
& Separado & 50 & 13,16 \\
& Viudo & 68 & 17,89 \\
\multirow{5}{*}{ Ocupación } & Empleado & 44 & 11,58 \\
& Desempleado & 36 & 9,47 \\
& Jubilado & 217 & 57,11 \\
& Jubilado y con trabajo & 83 & 21,84 \\
\hline
\end{tabular}

Tabla 2. Índice COPD en adultos mayores por sexo. Área Oriente, Santiago de Chile, años 2011-2012.

\begin{tabular}{lllcc}
\hline Grupo & Variable & Categoría & media & D.E. \\
\hline \multirow{4}{*}{ Hombres } & cariado & 1,35 & 1,87 \\
& Historia de & obturado & 8,51 & 639 \\
& caries & perdido & 11,71 & 7,93 \\
& & COPD & $\mathbf{2 1 , 5 8}$ & $\mathbf{4 , 8}$ \\
\multirow{4}{*}{ Mujeres } & cariado & 1,24 & 2,02 \\
& Historia de & obturado & 8,81 & 6,39 \\
& caries & perdido & 12,4 & 7,68 \\
& & COPD & $\mathbf{2 2 , 4 6}$ & $\mathbf{4 , 1 2}$ \\
\multirow{2}{*}{ Total } & & cariado & 1,28 & 1,97 \\
& Historia de & obturado & 8,71 & 6,11 \\
& caries & perdido & 12,16 & 7,76 \\
& & COPD & $\mathbf{2 2 , 1 6}$ & $\mathbf{4 , 4}$ \\
\hline
\end{tabular}

por caries. Este valor fue mayor en mujeres $(64,92 \%)$. La media de piezas remanentes en boca fue de 15,9 , siendo mayor en hombres. El índice COPD fue de 22,16 (IC: $95 \% 21,72 ; 22,60)$ sin presentar diferencias significativas por sexo $(p=0,96)$; sin embargo el valor del índice COPD aumenta con la edad $(\mathrm{p}<0.05)$. La media de piezas dentarias cariadas fue de 1,28 (IC: $95 \%$ 1,08; 1,48); obturadas 8,71(IC: $95 \% 8,09 ; 9,32)$ y perdidas 12,16 (IC: 95\% 11,38; 12,95) (Tabla 2).

Un 43\% clasificó con higiene oral regular y un $23,8 \%$ deficiente. La prevalencia de enfermedad periodontal fue de $97,6 \%$. Un 41,52\% de los adultos mayores presentaron tártaro supragingival y obturaciones defectuosas (código 2 del índice CPITN) siendo la necesidad de tratamiento periodontal, la tartrectomía, cambio de obturaciones y enseñanza de higiene oral. Un $17,37 \%$ presentó sacos periodontales de 3 a $4 \mathrm{~mm}$ (código 3 del índice CPITN) y un $19,74 \%$ sacos periodontales mayores a $5 \mathrm{~mm}$ (código 4 del índice CPITN). No se presentaron diferencias significativas por sexo $(p=0,006)$ ni por grupo de edad ( $\mathrm{p}=0,085)$ (Gráfico 1).

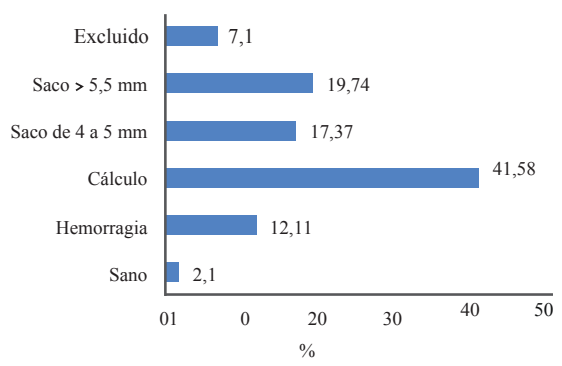

En cuanto a la situación protésica, un 59,5\% usa prótesis removible en el maxilar superior y un $40,3 \%$ en el inferior. Al estudiar el tipo de prótesis dental, se observó que para el maxilar superior, un $40,3 \%$ 
Tabla 3. Relación entre estado de salud oral y percepción de calidad de vida en adultos mayores. Área Oriente, Santiago de Chile, años 2011-2012.

\begin{tabular}{lcccc}
\hline Variable & GOHAI $^{* *}$ & Media & D.E & p \\
\hline cariado & 0 & 1,24 & 1,898 & 0,435 \\
& 1 & 1,43 & 2,194 & \\
obturado & 0 & 8,1 & 6,004 & $0,000^{*}$ \\
& 1 & 10,7 & 6,087 & \\
extraída & 0 & 12,97 & 7,77 & $0,001^{*}$ \\
& 1 & 9,7 & 7,428 & \\
COPD & 0 & 22,3 & 4,405 & 0,365 \\
& 1 & 21,82 & 4,326 & \\
dientes & 0 & 15,03 & 7,77 & $0,001^{*}$ \\
remanentes & 1 & 18,3 & 7,428 & \\
\hline
\end{tabular}

*valores de p inferiores a $0,05 . * *$ GOHAI calidad de vida: $0=$ mala / $1=$ buena)

usa prótesis parcial removible y un $19,2 \%$ prótesis total. En el maxilar inferior, el $32,1 \%$ usa prótesis parcial removible y un $8,2 \%$ prótesis total (Gráfico 2). En el 70\% de los casos, la prótesis removible es acrílica en el maxilar superior y un $55,5 \%$ en el maxilar inferior.

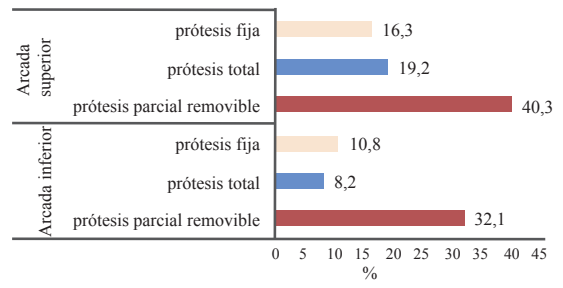

En relación a la necesidad de rehabilitación con prótesis removible, para el maxilar superior, el $34,2 \%$ necesita prótesis removible de tipo parcial y un $4,21 \%$ de tipo total. Para el maxilar inferior, un $57,4 \%$ necesita prótesis removible de tipo parcial y un $1,8 \%$ necesita prótesis total (Gráfico 3).

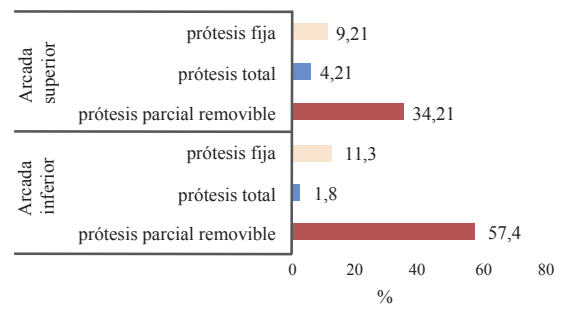

La calidad de vida relacionada con la salud oral fue de 48,15 (D.E. 9,83 ) de media del puntaje del índice GOHAI, en donde el valor mínimo fue de 15 y el máximo de 60. Según las categorías establecidas para este estudio, un $76,6 \%$ de los adultos mayores estudiados percibe como mala su calidad de vida relacionada con la salud oral (puntuación < 57), sin observarse diferencias estadísticamente significativas para las variables: sexo, edad, estado civil, nivel educacional y situación laboral. Sin embargo, estas diferencias son significativas para componente obturado y extraído del índice COPD y las variables relacionada con el uso, tipo y necesidad de prótesis removible $(\mathrm{p}<0,05)$.

\section{Discusión}

Son escasos los estudios epidemiológicos realizados en América Latina que relacionan el estado de salud oral con la calidad de vida en los adultos mayores. Específicamente en Chile, las referencias existentes están basadas en el estudio de Gamonal para el estado de salud oral (13) y en la Encuesta Na- cional de Salud y Calidad de vida en la vejez $(2,3)$.

A nivel mundial se observa que la salud bucal de los adultos mayores es en general deficiente, en donde se destacan como principales problemas, la pérdida de piezas dentarias, presencia de caries dental, elevada prevalencia de enfermedad periodontal, xerostomía y lesiones de la mucosa bucal $(14,15)$. Resulta pertinente referirse a la caries dental como una de las enfermedades bucales de mayor prevalencia y principal causa de pérdida de dientes al finalizar la etapa del ciclo vital. Los adultos mayores presentan en general entre 3,8 y 15,1 dientes remanentes en su boca y un $33 \%$ de edentulismo $(2,3,16,17)$.

En términos de historia de caries dental, los valores encontrados son similares a los reportados otros autores $(13,16,18,19)$. Más de la mitad de ellos presentaron lesiones cavitadas por caries dental sin tratar, siendo mayor en las mujeres. El índice COPD fue de 22,16 (IC:95\% 21,72-22,60) sin presentar diferencias significativas por sexo ( $p>0,05)$; sin embargo aumenta con la edad $(p<0,05)$. Este valor es levemente menor al compararlo con Gamonal (COPD: 26,02) y Arteaga (COPD: 24,9) para Chile y en países latinoamericanos como Brasil (COPD: 29,9) (20). En población europea los valores varían entre 22,2 a 30,2 (17, 21).

El diagnóstico de la enfermedad periodontal es posible de realizar después del minucioso procedimiento que realiza el especialista en periodoncia, sin embargo el índice CPITN diseñado con fines epidemiológicos y recomendado por la OMS se ha transformado en una excelente herramienta para realizar un examen periodontal básico e identificar quienes necesitan tratamiento 
(22). La prevalencia de enfermedad periodontal en los adultos mayores es elevada $(97,6 \%)$, siendo coincidentes con otras investigaciones $(13,23,24)$. Además, los hábitos de higiene oral regular o deficiente incrementan su avance y complejidad junto con la necesidad de derivación y tratamiento por especialista, elevando significativamente los costos.

La rehabilitación protésica en adultos mayores ha aumentado considerablemente en los últimos tiempos, encontrándose entre un $51,2-75,8 \%$ de ellos como portadores de algún tipo de prótesis dental, generalmente removibles y acrílicas. En cuanto a la necesidad de rehabilitación protésica, entre un 47,6 - 70,5\% de la población adulta mayor requiere de algún tipo de prótesis siendo mayores las necesidades para la arcada inferior. Estas cifras son concordantes con otras investigaciones $(2,3,25)$.

Las enfermedades orales influyen en la calidad de vida afectando diversos aspectos del diario vivir (26). La percepción de la salud oral es frecuentemente negativa en ésta población ya que tienden a aceptar las enfermedades bucales como consecuencia del envejecimiento. Sólo el 23,4\% percibió como buena su calidad de vida relacionada con la salud oral, valor inferior a lo observado por otros autores $(27,28,29,30)$.

El impacto de la calidad de vida relacionada con la salud oral se asoció significativamente al componente obturado, extraído del índice COPD, la media de dientes remanentes y el uso, tipo y estado de la prótesis removible $(\mathrm{p}<0.05)$, concordando con lo expuesto en otras investigaciones (Tabla 3).

Los problemas que afectan la salud bucal de los adultos mayores cobran importancia por el impacto que tienen en su calidad de vida, así como también por su complejidad, en donde se requiere de una atención odontológica de especialidad, la cual es escasa en el nivel primario de atención y costosa.

\section{Referencias Bibliográficas}

1. Ministerio de Salud de Chile. Encuesta Nacional de Salud. Santiago de Chile: Ministerio de Salud de Chile; 2003.

2. San Martín C, Villanueva J. Cambios sistémicos en el paciente adulto mayor. Revista Dental de Chile. 2002;93(2):11-3.

3. Pontificia Universidad Católica de Chile. Primera Encuesta Nacional de Calidad de Vida en la Vejez 2007.Santiago de Chile: Pontificia Universidad Católica de Chile; 2008. (Citado el 25 de Mayo de 2011). Disponible en: http:// www.uc.cl/sociologia/download/ encuesta_04 junio.pdf

4. Petersen PE. The World Oral Health Report 2003: continuous improvement of oral health in the 21st century--the approach of the WHO Global Oral Health Programme.. Community Dent Oral Epidemiol. 2003;31( S1):3-23.

5. Locker D. Self-esteem and socioeconomic disparities in selfperceived oral health. J Public Health Dent. 2009;69(1):1-8.

6. Locker D, Quiñonez, C. Functional and psychosocial impacts of oral disorders in Canadian adults: a national population survey. J Can Dent Assoc. 2009; 75(7):521a-521e.

7. Ministerio de Salud. Guía clínica: Salud Oral Integral para el Adulto de 60 años. Santiago de Chile, Chile: Ministerio de Salud; 2007.

8. Gamonal J, Mendoza C, Espinoza I, et al. Clinical attachment loss in Chilean adult population:
First Chilean National Dental Examination Survey. J Periodontol. 2010;81(10):1403-10.

9. Espinoza I, Rojas R, Aranda W, Gamonal J. Prevalence of oral mucosal lesions in elderly people in Santiago, Chile. J Oral Pathol Med. 2003;32(10):571-5.

10.Riera P, Martínez B. Morbidity and mortality for oral and pharyngeal cancer in Chile. Rev Med Chil. 2005;133(5):555-63.

11.Misrachi C, Lamadrid S. Salud Oral y Conductas Asociadas en Adultos Mayores de Bajos Recursos. Cuadernos Médico Sociales. 1997; 38(4):79-86.

12.Kidd E. Essentials of dental caries: The disease and its management. 3a ed. Oxford and New York: Oxford University Press; 2005.

13. Gamonal J. Prevalencia de enfermedades periodontales y de caries dental en la población de 35-44 y 65-74 años de nivel socioeconómico bajo y medio bajo de la Provincia de Santiago, Región Metropolitana y determinación de los recursos humanos necesarios para su tratamiento. Magíster en Periodontología. Santiago de Chile. Universidad de Chile, 1996.

14.Petersen PE, Yamamoto $T$. 2005. Improving the oral health of older people: the approach of the WHO Global Oral Health Programme. Community Dent Oral Epidemiol. 2005;33:81-92.

15.Turner MD, Ship JA. Dry mouth and its effects on the oral health of elderly people. J Am Dent Assoc. 2007;138:15S-20S.

16.Arteaga O, Urzua I. Espinoza I. Muñoz A. Mendoza C. Prevalencia de caries y pérdida de dientes en población de 65 a 74 años de Santiago Chile. Rev Clin Periodoncia Implantol Rehabil Oral. 2009;2(3):161-166. 
17.Bourgeois D, Nihtila A, Mersel A. Prevalence of caries and edentulousness among 65-74 year olds in Europe. Bull Word Health Organ. 1998;76(4):413-7.

18.Khalifa N, Allen PF, Abu-bakr $\mathrm{NH}$, Abdel-Rahman ME, Abdelghafar KO. A survey of oral health in a Sudanese population. BMC Oral Health 2012;12(5):1-9

19.Lundgren M, Emilson CG, Osterberg T. 1996. Caries prevalence and salivary and microbial conditions in 88 year-old Swedish dentate people. Acta Odontol Scand. 1996 Jun;54(3):193-9.

20.Haikal DS, Paula AM, Martins AM, Moreira AN, Ferreira EF. Self-perception of oral health and impact on quality of life among the elderly: a quantitative-qualitative approach. Cien Saude Colet. 2011 Jul;16(7):3317-29.

21.Berg R, Berkey DB, Tang JM, Baine C, Altman DS. Oral health status of older adults in Arizona: results from the Arizona Elder Study. Spec Care Dentist. 2000;20(6):226-33.
22.Bassani DG, da Silva CM, Oppermann RV. Validity of Community Periodontal Index of Treatment Needs (CPITN) for population periodontitis screening. Cad Saude Publica. 2006 Feb;22(2):277-83.

23.Rojas González C, Segovia Chamorro J, Raccoursier Schwerter V, Godoy Olave J, Lopetegui Buschmann MS. Estado Periodontal y Necesidad de Tratamiento en Pacientes GES 60 Años de Villa Alemana. Rev. Clin. Periodoncia Implantol. Rehabil Oral. 2010;3(2):86-89.

24.Rola Al Habashneh, Yousef S. Khader, Shatha Salameh. Use of the Arabic version of Oral Health Impact Profile-14 to evaluate the impact of periodontal disease on oral health-related quality of life among Jordanian adults. J Oral Sci. 2012;54(1):113-20.

25.Llodra J, Bravo M, Cortés F. Encuesta de Salud Oral en España (2000). RCOE. 2002;7:19-63.

26. Gift HC, Redford M. Oral health and the quality of life. Clin Geriatr Med. 1992;8:673-83.
27.Zuluaga DJ, Montoya JA, Contreras CI, Herrera RR. Association between oral health, cognitive impairment and oral health-related quality of life. Gerodontology. 2012; 29(2):667-73.

28. Biazevic MG, Rissotto RR, Michel-Crosato E, Mendes LA, Mendes MO.. Relationship between oral health and its impact on quality of life among adolescents. Braz Oral Res. 2008;22 (1):36-42.

29. Kshetrimayum N, Reddy CV, Siddhana S, Manjunath M, Rudraswamy S, Sulavai S. Oral health-related quality of life and nutritional status of institutionalized elderly population aged 60 years and above in Mysore City, India. Gerodontology. 2013;30(2):119-25.

30.Zhao L, Lin HC, Lo EC, Wong MC. Clinical and socio-demographic factors influencing the oral health-related quality of life of Chinese elders. Community dent health. 2011;28(3):206-10. 